\title{
Solution Growth of Well-Aligned ZnO Nanorods on Sapphire Substrate
}

\author{
G.-Z. JIA ${ }^{a, *}$, B.-X. HAO ${ }^{b}$, X.-C. LU ${ }^{b}$, X.-L. WANG ${ }^{b}$, Y.-M. $\mathrm{LI}^{b}$, AND J.-H. YAO \\ ${ }^{a}$ Tianjin Institute of Urban Construction, Tianjin 300384, P.R. China
}

${ }^{b}$ The Key Lab of Advanced Technique and Fabrication for Weak-Light Nonlinear Photonics Materials Ministry of Education, TEDA Applied Physics School, Nankai University, Tianjin 300475, P.R. China

(Received December 18, 2012; in final form April 11, 2013)

\begin{abstract}
Vertically well-aligned $\mathrm{ZnO}$ nanorods arrays were synthesized on sapphire substrates by chemical bath deposition. Those sapphire substrates were seeded to control the density and orientation of ZnO nanorods using sol-gel method. Well-aligned and uniformly distributed $\mathrm{ZnO}$ nanorods in a large scale were obtained with strongly (002) preferential orientation. The structural properties were characterized by X-ray diffraction spectrometer and morphological characteristics were analyzed by scanning electron microscopy, respectively. The $\mathrm{ZnO}$ nanorods are obvious hexangular wurtzite structure and preferentially oriented along the $c$-axis (002) and growth vertically to the substrates. The optical properties were further thoroughly studied. What is more, the influences of the strain between substrate and $\mathrm{ZnO}$ nanorods due to thickness of the $\mathrm{ZnO}$ seed-layer on the characteristics and optical properties of $\mathrm{ZnO}$ were also analyzed.
\end{abstract}

DOI: $10.12693 /$ APhysPolA.124.74

PACS: 68.37.Yz, 78.40.Fy, 68.55.J-

\section{Introduction}

Owing to its wide band gap energy of $3.37 \mathrm{eV}$ and large exciton binding energy of $60 \mathrm{meV}$ at room temperature, zinc oxide has been one of the most important semiconducting materials [1-3]. ZnO crystals with different morphologies including nanorods, nanotube, nanoflower, nanopins and other novel structures have been successfully prepared. Recently, well-aligned $\mathrm{ZnO}$ nanowire or nanorods have attracted an extensive attention because of its potential application in photonic, electronic, optoelectronic and electrochemical nanodevices. Many techniques have been reported to prepare well-aligned nanostructure arrays involve aqueous solution method, molecular beam epitaxy, metal organic chemical vapor deposition, template method, and so forth. Various theories about the nanostructure have also been investigated in detail [4-6].

Among all the methods of preparing $\mathrm{ZnO}$ nanorods, chemical bath deposition (CBD) is much more attractive because of its excellent characteristics. It is simple, cost-effective, can be controlled easily and could be carried out at low temperature; in addition, chemical bath deposition has become a perfect candidate for large-scale production of nanostructure materials $[1,2,7-10]$. We have studied the morphological change of $\mathrm{ZnO}$ nanostructure grown by chemical bath deposition [10]. The single-crystalline $\mathrm{ZnO}$ films on $\mathrm{ZnO}$-buffered $a$-plane sapphire successfully prepared by chemical bath deposition and a reciprocal space map indicated that the lattice parameters of the $\mathrm{ZnO}$ film were very close to the wurtzite-type $\mathrm{ZnO}$ [11]. In addition it is well known that substrate materials are very important for the fabrication of photoelec-

*corresponding author; e-mail: gzjia@semi.ac.cn tric devices. Among all the substrate materials, sapphire substrate has its unique advantages, such as mature production technology, high-crystalline perfection and high stability, especially, $\mathrm{ZnO}$ can be prepared epitaxially on the sapphire and has a good flatness, which is beneficial to the production of the photoelectric devices.

In this paper, vertically well-aligned $\mathrm{ZnO}$ nanorods arrays on sapphire substrates were prepared by chemical bath deposition with considering the effect of seed layer. The density and orientation of $\mathrm{ZnO}$ nanorods can be controlled by seed-layer. The structural and optical properties of as-grown $\mathrm{ZnO}$ nanorods on sapphire were investigated by X-ray diffraction (XRD), photoluminescence (PL) and Raman spectrum. The influences of the strain between substrate and $\mathrm{ZnO}$ nanorods due to thickness of the $\mathrm{ZnO}$ seed-layer on the characteristics and optical properties of $\mathrm{ZnO}$ were further analyzed.

\section{Experiments}

All chemicals used in this experiment, such as zinc acetate dihydrate $\mathrm{Zn}(\mathrm{Ac})_{2} \cdot 2 \mathrm{H}_{2} \mathrm{O}$, hexamethylenetetramine $\left(\mathrm{C}_{6} \mathrm{H}_{12} \mathrm{~N}_{4}\right)$, 2-methoxyethanol, diethanol amine (DEA) are analytical reagents and used as purchased without further purification. The well-aligned $\mathrm{ZnO}$ nanorods arrays are synthesized at low temperature combining two-step chemical solution method.

The first step was coating the $\mathrm{ZnO}$ seed on sapphire substrate by a sol-gel method. The gel-sol was prepared by dissolving $\mathrm{Zn}\left(\mathrm{CH}_{3} \mathrm{COO}\right)_{2} \cdot 2 \mathrm{H}_{2} \mathrm{O}$ into the 2-methoxyethanol under mild magnet stirring and then adding the DEA slowly in the above-mentioned solutions drop by drop as a sol stabilizer. After stirring at $60^{\circ} \mathrm{C}$ for $2 \mathrm{~h}$ and aging for $24 \mathrm{~h}$, a transparent and homogeneous sol was formed. Subsequently, the sol was coated on the sapphire substrates by pulling method at the suitable experimental conditions. The above-mentioned process was 
repeated for several times and the resulting films were annealed at $500{ }^{\circ} \mathrm{C}$ for $1 \mathrm{~h}$ to form the $\mathrm{ZnO}$ film. The second step was the formation of $\mathrm{ZnO}$ nanorods arrays on the as-pretreated sapphire substrate by chemical bath deposition. The detailed experiment process was the same as that of the paper reported by Jia et al. [1, 2, 10]. The as-grown films were rinsed with deionized water for several times and dried in air at room temperature before characterization.

The as-prepared $\mathrm{ZnO}$ nanorods were characterized by Rigaku X-ray diffractometer using $\mathrm{Cu} K_{\alpha}$ radiation $(\lambda=$ $1.54178 \AA$. The diffraction angle was scanned from $20^{\circ}$ to $80^{\circ}$ at the scanning speed of $0.02^{\circ}$ per second. The morphology properties were studied by SHIMADZU SS550 scanning electron microscopy (SEM), the room temprature PL spectroscopy was measured using Edinburgh FSP920 fluorescence spectrometer with the Xe lamp as the excitation light source and excitation wavelength was $325 \mathrm{~nm}$. The vibrational properties were investigated by the Raman spectra recorded on Renishaw Raman spectrometer with $514 \mathrm{~nm}$ as the excitation wavelength.

\section{Results and discussion}

Figure 1a-d shows the SEM of $\mathrm{ZnO}$ nanorods arrays with different thickness of seed-layer on sapphire substrate by chemical bath deposition: (a) one seed-layer, (b) three seed-layer, (c) five seed-layer, (d) seven seed-layer, respectively. It indicates that all the as-grown $\mathrm{ZnO}$ nanorods are hexagonal facets and aligned preferentially along the $c$-axis direction. It can be seen from the cross-section of the $\mathrm{ZnO}$ nanorods that all the nanorods on sapphire substrate have the same height. However, those nanorods have the different diameters on substrates with different seed-layer thickness. Lee et al. reported that the density, size and orientation of as-grown nanorods are mainly dependent on the $\mathrm{ZnO}$ seed layer [12] which plays a remarkable role in formation of well-aligned and uniformly distributed $\mathrm{ZnO}$ nanorods. On the one hand, the lattice constants of the $\mathrm{ZnO}$ seeds are matching with that of the $\mathrm{ZnO}$ nanorods; on the other hand, the $\mathrm{ZnO}$ seed-layer provides the nucleation sites for the growth of $\mathrm{ZnO}$ nanorods which thereby decreases the nucleation barrier by decreasing the interface energy and makes the $\mathrm{ZnO}$ nanorods easy to grow [13].

Figure 2 shows the XRD patterns of $\mathrm{ZnO}$ nanorods arrays with different thickness of seed-layer on sapphire substrate by chemical bath deposition:(a) one seed-layer, (b) three seed-layer, (c) five seed-layer, (d) seven seed-layer, respectively. It can be seen that all the $\mathrm{ZnO}$ nanorods deposited on different thickness seed-layer have the strongest (002) diffraction peak and narrow FWHM well indexed to the Bragg reflections of the standard wurtzite structure zinc oxide (JCPDS Card File No. 36$-1451, a=0.3249 \mathrm{~nm}$ and $c=0.5206 \mathrm{~nm}$ ) [14], indicating that these $\mathrm{ZnO}$ nanorods have good crystallinity and perfectly oriented perpendicular to the substrate, which can be obviously seen from the SEM images. What is more, compared with the X-ray diffractograms of seed
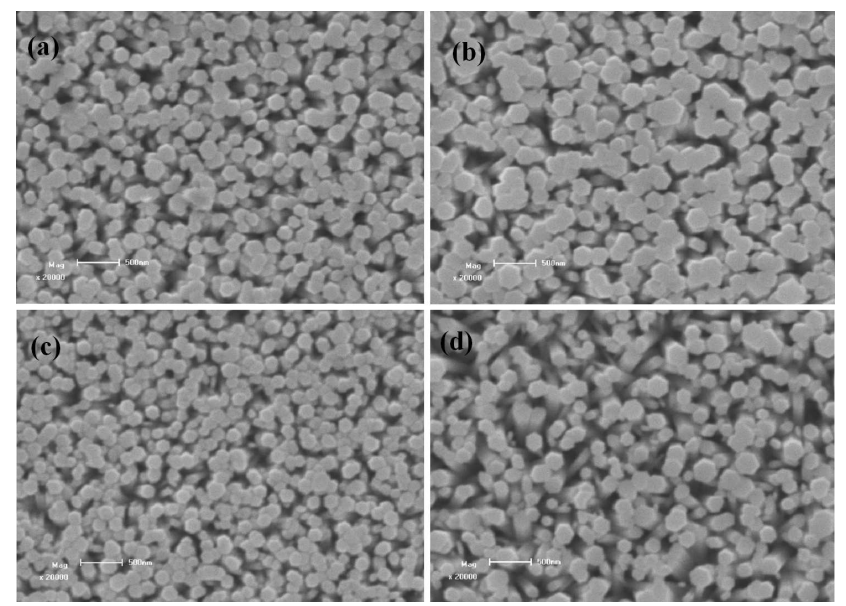

Fig. 1. SEM of $\mathrm{ZnO}$ nanorods arrays with different thickness of seed-layer on sapphire substrate by chemical bath deposition. (a) one seed-layer, (b) three seed-layer, (c) five seed-layer, (d) seven seed-layer, respectively.

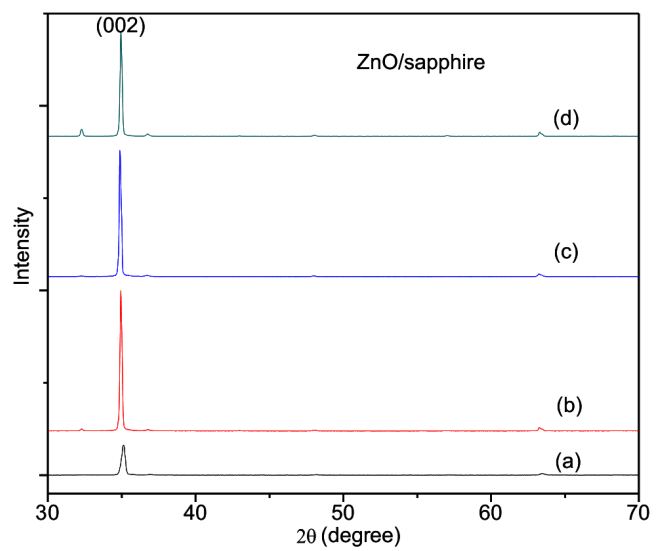

Fig. 2. XRD patterns of $\mathrm{ZnO}$ nanorods arrays with different thickness of seed-layer on sapphire substrate by chemical bath deposition. (a) one seed-layer, (b) three seed-layer, (c) five seed-layer, (d) seven seed-layer, respectively.

free $\mathrm{ZnO}$ power, the intensity of other diffraction peaks of samples in our experiment is much weaker than that of (002) diffraction peak, suggesting that the $\mathrm{ZnO}$ seed-layer plays a very important role on the growth orientation of $\mathrm{ZnO}$ nanorods. However, the interface strain among the substrate, $\mathrm{ZnO}$ seed-layer and $\mathrm{ZnO}$ nanorods should be taken into consideration. The lattice constants $a$ and $c$ of wurtzite structure zinc oxide are calculated according to Bragg's law (1) and Eq. (2) which is related with plane spacing and Miller indices [15]:

$2 d \sin \theta=n \lambda$,

where $d$ is the plane spacing, $n$ is the order of diffraction that usually is $1, \lambda$ is $\mathrm{X}$-ray wavelength.

$$
\frac{1}{d_{(h k l)}^{2}}=\frac{4}{3} \frac{h^{2}+h k+k^{2}}{a^{2}}+\frac{l^{2}}{c^{2}},
$$

where $d$ is the plane spacing, $h, k, l$ is the Miller indices. 
Combining the above equations, the calculation results are summarized in Table.

TABLE

The Lattice constants of $\mathrm{ZnO}$ nanorods and its strain among the interfaces along $\mathrm{c}$ axis deposited on the different thickness seed-layer on sapphire substrates.

\begin{tabular}{c|c|c}
\hline \hline Seed-layer & Lattice constants [nm] & Strain, $\varepsilon_{z z}[\%]$ \\
\hline one & $a=0.3207, c=0.5106$ & -1.92 \\
three & $a=0.3204, c=0.5131$ & -1.44 \\
five & $a=0.3221, c=0.5139$ & -1.28 \\
seven & $a=0.3220, c=0.5130$ & -1.45
\end{tabular}

The strain $\left(\varepsilon_{z z}\right)$ among the interfaces is obtained from the following relation (3) [16] and the results are also shown in Table.

$$
\varepsilon_{z z}=\left(c-c_{0}\right) / c_{0} \times 100 \%,
$$

where $c$ is the calculation value of lattice constants according to the X-ray diffractograms while $c_{0}$ is the unstrained lattice constant of standard wurtzite structure $\mathrm{ZnO}$. As is shown in Table, the strain among the substrate, $\mathrm{ZnO}$ seed-layer and the $\mathrm{ZnO}$ nanorods is the compressive strain due to the strain value $\varepsilon_{z z}$ is negative, corresponding to the smaller lattice constants than the standard wurtzite structure $\mathrm{ZnO}$.

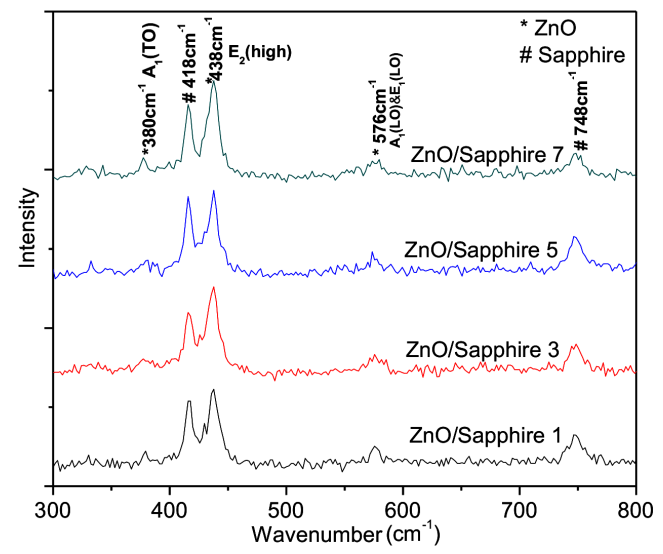

Fig. 3. Raman optical spectrum of $\mathrm{ZnO}$ nanorods arrays with different thickness of seed-layer on sapphire substrate by chemical bath deposition.

Hexagonal wurtzite structure $\mathrm{ZnO}$ belongs to the space group $C_{6 \nu}^{4}$. Group theory predicts that single-crystalline $\mathrm{ZnO}$ has eight sets of optical phonon modes at $\Gamma$ point of the Brillouin zone, summarized as $A_{1}+E_{1}+2 E_{2}+$ $2 B_{1}$, where $A_{1}, E_{1}$ and $E_{2}$ modes are the Raman active phonons while $A_{1}$ and $E_{1}$ modes are also infrared active, $B_{1}$ (low) and $B_{1}$ (high) modes are usually silent. What is more, $A_{1}$ and $E_{1}$ modes belong to polar symmetries and are split into longitudinal (LO) and transverse (TO) optical components [15, 17].

Figure 3 shows the Raman optical spectrum of as-synthesized $\mathrm{ZnO}$ nanorods arrays. The remarkable $E_{2}$ (high) mode located at $438 \mathrm{~cm}^{-1}$ indicates the charac- teristic of wurtzite structure $\mathrm{ZnO}$. The weak scattering peaks at $380 \mathrm{~cm}^{-1}$ and $576 \mathrm{~cm}^{-1}$ correspond to $A_{1}$ (TO), and $A_{1}(\mathrm{LO})$ and $E_{1}(\mathrm{LO})$, respectively, which are related to intrinsic defects such as oxygen vacancy, zinc interstitial, and so on [18], the peak at $331 \mathrm{~cm}^{-1}$ is the second-order Raman scattering peak. The intensity of those peaks is much weaker than that of the $E_{2}$ modes, suggesting that $\mathrm{ZnO}$ nanorods prepared by chemical bath deposition with the assistance of $\mathrm{ZnO}$ seed-layer have small defects. The scattering peaks located at $418 \mathrm{~cm}^{-1}$ and $748 \mathrm{~cm}^{-1}$ are also observed, associated with the vibrational properties of sapphire [19-21]. The intensity of $E_{2}$ (high) peak increases with increasing the thickness of $\mathrm{ZnO}$ seed-layer, confirming that the $\mathrm{ZnO}$ nanorods have better crystallinity deposited on the thicker $\mathrm{ZnO}$ seed-layer, the result is in accordance with the XRD dada analysis. $E_{2}$ (high) is sensitive to the strain and the frequency shift of $E_{2}$ (high) peak results from the strain variation [18]. By contrast to the $437 \mathrm{~cm}^{-1}$ of the bulk $\mathrm{ZnO}$, all the $E_{2}$ (high) peaks of $\mathrm{ZnO}$ nanorods grown in our experiment have slight blueshift, indicating there are compressive strain [20,22] among the substrates, $\mathrm{ZnO}$ seed-layer and $\mathrm{ZnO}$ nanorods corresponding to the strain $\varepsilon_{z z}$ calculated in Table. Moreover, as the thickness of $\mathrm{ZnO}$ seed-layer increases, the frequency shift decreases, which means interface strain decreases, thus the $\mathrm{ZnO}$ nanorods have the better crystallinity.

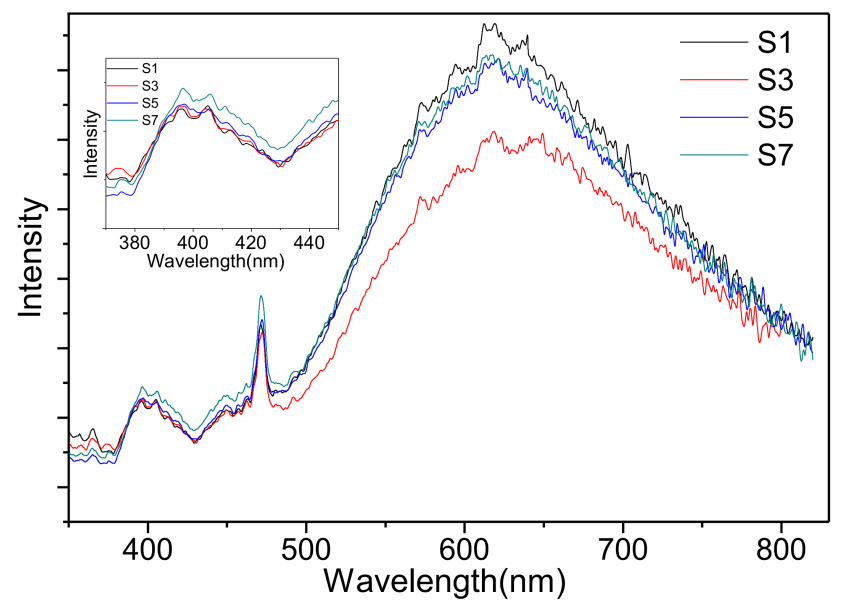

Fig. 4. Room temperature photoluminescence spectrum of $\mathrm{ZnO}$ nanorods arrays with different thickness of seed-layer on sapphire substrate by chemical bath deposition.

Figure 4 shows the room temperature photoluminescence (PL) spectra of as-grown $\mathrm{ZnO}$ nanorods on sapphire carried out with $325 \mathrm{~nm}$ as the excitation source. All the $\mathrm{ZnO}$ nanorods arrays samples show a typical UV emission peak, a broad visible emission band and a sharp peak centered at $471 \mathrm{~nm}$. The UV emission corresponding to the near-band-edge (NBE) emission, ranging from $380 \mathrm{~nm}$ to $430 \mathrm{~nm}$ and the central value located at $395 \mathrm{~nm}$ $(3.14 \mathrm{eV})$, is usually ascribed to the radiative recombination of $\mathrm{ZnO}$ free excitons [23]. It is well known that the 
low temperature hydrothermal methods of preparing the $\mathrm{ZnO}$ crystallinity are very easy to introduce the defects such as oxygen vacancy, zinc vacancy, oxygen interstitial and so forth, which is in agreement with the Raman scattering results. These defects can weaken the near-band-edge (NBE) light emission intensity of $\mathrm{ZnO}$ as a nonradiative center $[20,24]$. The broad visible emission band from $490 \mathrm{~nm}$ to $800 \mathrm{~nm}$ as well as central value around $620 \mathrm{~nm}$ is closely related to the defects of $\mathrm{ZnO}$. Lo et al. indicated that the visible emission originated from the recombination of the photogenerated hole with the single ionized charge state of the $\mathrm{ZnO}$ defects [20, 21, 25-28]. Zhou et al. reported that the oxygen vacancy was not the direct reason of yellow-green emission [25] while it may be attributed to the oxygen interstitial defects [2]. However, the accurate reason remains unclear and we need to further analyze. In addition, the sharp peak at $471 \mathrm{~nm}$ results from the xenon lamp spectral line.

The inset pattern is the UV emission band of the as-grown $\mathrm{ZnO}$ nanorods samples. It can be clearly seen that the UV emission intensity increases as the nanorods diameter increases, which is possibly attributed to the good crystallinity for the large nanorods diameter. In addition, the visible emission intensity slightly decreases as the nanorods diameter increases, which indicates that the thinner seed-layer results in the larger nanorods diameter and thereby introduces fewer defects.

\section{Conclusion}

Vertically well-aligned $\mathrm{ZnO}$ nanorods arrays were synthesized on sapphire substrates combining the sol-gel method with chemical bath deposition (CBD). The thin $\mathrm{ZnO}$ seed-layer was deposited on the sapphire substrate to control the density and orientation of $\mathrm{ZnO}$ nanorods. The results showed that the $\mathrm{ZnO}$ nanorods were hexangular wurtzite structure and had good crystallinity as well as preferentially oriented along the $c$-axis by XRD, PL, and Raman spectrum. There existed small compressive strain among the substrate, $\mathrm{ZnO}$ seed-layer and $\mathrm{ZnO}$ nanorods and the compressive strain decreases with the thickness of the $\mathrm{ZnO}$ seed-layer increases.

\section{Acknowledgments}

This work has been partly supported by the National Key Basic Research Program of China (2012CB934201), the National Natural Science Foundation of China (11147024, 11247025, and 11074129).

\section{References}

[1] N. Hongsith, S. Choopun, J.C. Mai, Science 37, 48 (2010).

[2] H.K. Liang, S.F. Yu, H.Y. Yang, Appl. Phys. Lett. 96, 101116 (2010).

[3] O. Lupan, T. Pauporte, B. Viana, I.M. Tiginyanu, V.V. Ursaki, R. Cortes, Appl. Mater. Inter. 2, 2083 (2010).

[4] Z. Guo, H. Zhang, D.X. Zhao, Y.C. Liu, B. Yao, B.H. Li, Z.Z. Zhang, D.Z. Shen, Appl. Phys. Lett. 97, 173508 (2010).
[5] M. Suchea, S. Christoulakis, K. Moschovis, N. Katsarakis, G. Kiriakidis, Thin Solid Films 515, 551 (2006).

[6] M.S. Kim, K.G. Yim, S. Kim, G. Nam, Lee, D.-Y. Kim, Jin Soo, Kim, Jong Su, J.-Y. Leem, Acta Phys. Pol. A 121, 217 (2012).

[7] G.W. Cong, H.Y. Wei, P.F. Zhang, W.Q. Peng, J.J. Wu, X.L. Liu, C.M. Jiao, W.G. Hu, Q.S. Zhu, Z.G. Wang, Appl. Phys. Lett. 87, 231903 (2005).

[8] S. Xu, Y.G. Wei, M. Kitkhsm, J. Liu, W.J. Mai, D. Davidovic, R.L. Snyder, Z.L. Wang, J. Am. Chem. Soc. 130, 14958 (2008).

[9] Q.X. Zhao, L.L. Yang, M. Willander, B.E. Sernelius, P.O. Holtz, J. Appl. Phys. 104, 073526 (2008).

[10] G.Z. Jia, Y.F. Wang, J.H. Yao, J. Ovonic. Res. 6, 303 (2010).

[11] T. Hamada, A. Ito, E. Fujii, D. Chu, K. Kato, Y. Masuda, J. Cryst. Growth 311, 3687 (2009).

[12] S.D. Lee, Y.S. Kim, M.S. Yi, J.Y. Choi, S.W. Kim, J. Phys. Chem. C 113, 8954 (2009).

[13] H. Zhang, D.R. Yang, X.Y. Ma, N. Du, J.B. Wu, D.L. Que, J. Phys. Chem. B 10, 827 (2006).

[14] J.J. Qiu, X.M. Li, W.Z. He, S.J. Park, H.K. Kim, Y.H. Hwang, J.H. Lee, Y.D. Kim, Nanotechnology 20, 155603 (2009).

[15] Q. Ahsanulhaq, S.H. Kim, Y.B. Hahn, J. Phys. Chem. Solids 70, 627 (2009).

[16] J. Tang, X.R. Yang, Mater. Lett. 60, 3487 (2006).

[17] J.H. Jang, J.H. Park, S.G. Oh, J. Ceram. Process. Res. 10, 783 (2009).

[18] A. Umar, C. Ribeiro, A. Al-Hajry, Y. Masuda, Y.B. Hahn, J. Phys. Chem. C 113, 14715 (2009).

[19] H. Verma, D. Mukherjee, S. Witanachchi, P. Mukherjee, M. Batzill, J. Cryst. Growth 312, 2012 (2010).

[20] G.Z. Jia, Y.F. Wang, J.H. Yao, J. Phys. Chem. Solids 73, 495 (2012).

[21] G.Z. Jia, Y.F. Wang, J.H. Yao, Digest J. Nanomater. Biostruct. 7, 261 (2012).

[22] O. Lupan, L. Chow, L.K. Ono, B.R. Cuenya, G.Y. Chai, H. Khallaf, S. Park, A. Schulte, J. Phys. Chem. C 114, 12401 (2010).

[23] R. Ghosh, D. Basak, S. Fujihara, J. Appl. Phys. 96, 2689 (2004).

[24] Y. Zhang, H.B. Jia, R.M. Wang, C.P. Chen, X.H. Luo, D.P. Yu, C. Lee, Appl. Phys. Lett. 83, 4631 (2003).

[25] C.X. Xu, G.P. Zhu, X. Li, Y. Yang, S.T. Tan, X.W. Sun, C. Lincoln, T.A. Smith, J. Appl. Phys. 103, 094303 (2008).

[26] G.W. Cong, H.Y. Wei, P.F. Zhang, W.Q. Peng, J.J. Wu, X.L. Liu, C.M. Jiao, W.G. Hu, Q.S. Zhu, Z.G. Wang, Appl. Phys. Lett. 87, 231903 (2005).

[27] S.S. Lo, D. Huang, Langmuir 26, 6762 (2010).

[28] H.L. Zhou, P.G. Shao, S.J. Chua, J.A. van Kan, A.A. Bettiol, T. Osipowicz, K.F. Ooi, G.K.L. Goh, F. Watt, Cryst. Growth. Des. 8, 4445 (2008). 